\title{
Evaluation of the Cox Equation to Derive Dynamic Contact Angle at Nanopores Imbibition: A Molecular Dynamics Study
}

\author{
S. Ahadian* and Y. Kawazoe \\ Institute for Materials Research (IMR), Tohoku University, Sendai 980-8577, Japan
}

\begin{abstract}
Molecular dynamics (MD) simulation is employed to simulate the imbibition of a designed nanopore by a fluid. The fluid is considered as a simple Lennard-Jones (LJ) fluid. For this system (i.e., LJ fluid and nanopore), the length of imbibition as a function of time for various interactions between the fluid and the pore wall is recorded. In almost all cases, the kinetics of imbibition is successfully described with the Lucas-Washburn (LW) equation. However, the deviation from the LW equation is observed in some cases. This nonconformity is contributed to the neglecting of the dynamic contact angle (DCA) in the LW equation. A hydrodynamic model (i.e., the Cox equation) is taken into consideration to calculate the DCA. It is demonstrated that the LW equation together with the Cox equation is able to justify the simulation results for those cases, which are not in good agreement with the simple LW equation. Further investigation on the MD simulation data reveals that the Cox equation is only appropriate to derive the DCA at small capillary numbers. This finding is in consonance with the theoretical background of this equation as well as experimental work. [doi:10.2320/matertrans.MRA2008405]
\end{abstract}

(Received October 28, 2008; Accepted February 27, 2009; Published April 22, 2009)

Keywords: nanopores imbibition, molecular dynamics simulation, Lucas-Washburn equation, dynamic contact angle, Cox equation

\section{Introduction}

Wetting of porous media is of great importance in many practical applications, such as oil recovery, civil engineering, dyeing of textile fabrics, ink printing, and also in new frontier technologies such as design of nanofluidic devices. Porous systems are usually complicated to model in detail. To characterize them, one approach is to measure the rate of penetration of a liquid into the medium, which is then modeled as a bundle of uniform capillaries. On macroscopic scale, a commonly used equation (i.e., the Lucas-Washburn (LW) equation) of such flow processes has been proposed almost a century ago. ${ }^{1,2)}$ The LW equation relates the length of liquid penetration in a straight-line capillary $H$ to the permeation time $t$, the capillary radius $R$, the viscosity and surface tension of the liquid $\eta$ and $\gamma_{\mathrm{lv}}$, respectively, and the contact angle between the liquid and the capillary wall $\theta$ as follows:

$$
H^{2}=\left(\frac{R \gamma_{\mathrm{lv}} \cos \theta}{2 \eta}\right) t
$$

The applicability of the LW equation has been tested for modeling liquid flow in nanopores. ${ }^{3,4)}$ To derive this equation, it is assumed that the contact angle does not change during the imbibition process. However, this assumption is not correct in general. Since the contact angle is corresponding to a moving wetting line and its value therefore depends on the wetting-line rate. ${ }^{5)}$ Hence, the modification of the LW equation is essential to take account a velocity-dependent dynamic contact angle. To estimate the dynamic contact angle (DCA), one can use one of various models of moving wetting lines, based on hydrodynamics, ${ }^{6,7)}$ molecular-kinetics ${ }^{8,9)}$ or phenomenology, ${ }^{10)}$ in which DCA is a function of wetting line velocity (i.e., $d H / d t$ ). Among these models to derive DCA, the Cox equation, ${ }^{6)}$ which is based on hydrodynamics, has the simplest form. Moreover, it has been

*Corresponding author, E-mail: ahadian@imr.edu, samad_ahadian@ yahoo.com shown ${ }^{11,12)}$ that for the relevant ranges of velocities and contact angles, the Cox correlation agrees with experimental data. Therefore, the Cox equation is used in the present investigation, which is given by the following expression:

$$
\theta_{d}=\left(\theta_{e}^{3}+9 A \frac{\eta}{\gamma_{\mathrm{lv}}} \frac{d H}{d t}\right)^{1 / 3}
$$

and $A=\ln (R / s)$, where $R$ is the characteristic length of the system, $s$ is the slip length, $\theta_{d}$ and $\theta_{e}$ denote the dynamic and equilibrium contact angles, respectively. In this case study (i.e., capillary penetration phenomenon), the characteristic length $R$ is the capillary radius. The slip length $s$ is the distance from the capillary wall that defines a region where the continuum description of fluid motion tends to break down. ${ }^{13)}$ Nevertheless, it is difficult to exactly calculate the value of the parameter $A$, but a rough approximation gives a value of about $14 .^{14)}$

The modified version of the LW equation including the effect of the DCA on the penetration kinetics is as follows:

$$
\frac{d H}{d t}=\left(\frac{R \gamma_{\mathrm{lv}} \cos \theta_{d}}{4 \eta H}\right)
$$

Recently, a general model to demonstrate fluid flow in nanopores has been proposed by Dimitrov et al. ${ }^{15)}$ This model is based on molecular dynamics (MD) simulation. They showed that the LW equation is a reliable approach to explain the kinetics of fluid penetration through nanopores. As stated before, this corollary is not true in general and one should take into account the influence of the DCA to draw a more realistic picture of this phenomenon. In what follows, we would like to show this fact by the aid of the MD simulation code developed by Dimitrov et al. ${ }^{15}$ ) Then, the applicability of the Cox equation to derive the DCA is put to test.

\section{Model and MD Simulation Description}

The snapshot of our model is illustrated in Fig. 1. It is comprised of a cylindrical nanotube with the radius $R=10$. The capillary wall is presented by the atoms of a triangular 


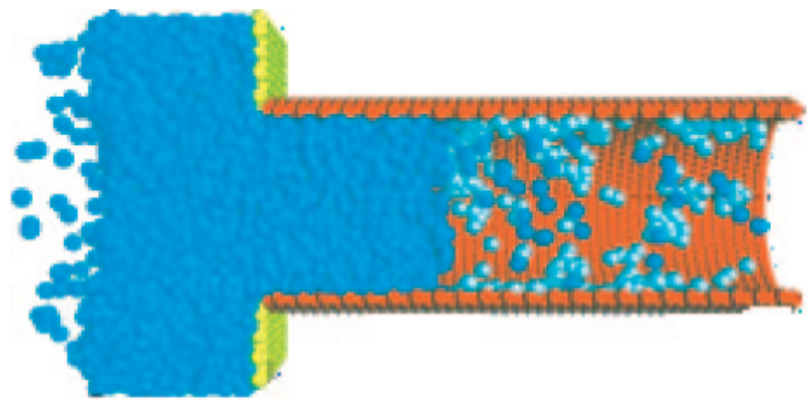

Fig. 1 An illustration of wetting of a designed nanopore by a LennardJones fluid. (Note that the fluid particles, the tube wall, and the atoms of the reservoir adhesive wall are showed in blue, brown and yellow, respectively.)

lattice, which has a lattice constant 1.0 in units of the fluid atom diameter $\sigma$. The right side of the capillary tube is closed by a hypothetically impenetrable wall, which holds the fluid particles inside the tube. The left side of the capillary tube is attached to a rectangular reservoir $40 \times 40$ involving fluid particles with periodic boundaries perpendicular to the tube axis. We shall say that to avoid entering the fluid particles into the tube before commencing the MD simulation runs, the capillary wall is taken into account completely hydrophobic. Therefore, the fluid particles stay in the reservoir as a stable fluid film. At the time $t=0$, set to be the onset of capillary filling, we switch the hydrophobic wall-fluid interactions into hydrophilic ones and then the fluid enters the tube. At the same time, we start to measure the structural and kinetic properties of the imbibition process at equal intervals of the time. In all simulation runs, we use a maximum capillary length $H_{\max }=55$.

MD simulation is a technique where the time evolution of a set of interacting particles is followed by interacting their equations of motion, which are notably based on the Newton law of motion. In the following, let us explain about intermolecular potentials that cause such kind of motion. The atoms of the capillary wall can fluctuate around their equilibrium positions at $R+\sigma$, corresponding to a finitely extensible nonlinear elastic potential (i.e., $U_{\mathrm{FENE}}$ ) as follows:

$$
U_{\mathrm{FENE}}=-15 \varepsilon_{\mathrm{w}} R_{0}^{2} \ln \left(1-\frac{r^{2}}{R_{0}^{2}}\right)
$$

where $\varepsilon_{\mathrm{w}}$ is the depth of the potential well, $R_{0}$ is a constant $\left(R_{0}=1.5\right)$ and $r$ is the distance between any pair of wall atoms. In the above equation, $\varepsilon_{\mathrm{w}}$ is defined as:

$$
\varepsilon_{\mathrm{W}}=1.0 k_{\mathrm{B}} T
$$

where $k_{\mathrm{B}}$ stands for the Boltzmann constant and $T$ is the temperature of the system. In addition, it is supposed that the wall atoms interact by a Lennard-Jones (LJ) potential as follows:

$$
U_{\mathrm{LJ}}(r)=4 \varepsilon_{\mathrm{ww}}\left[\left(\frac{\sigma_{\mathrm{ww}}}{r}\right)^{12}-\left(\frac{\sigma_{\mathrm{ww}}}{r}\right)^{6}\right]
$$

where $\varepsilon_{\mathrm{ww}}$ and $\sigma_{\mathrm{ww}}$ are the depth of the potential well and the effective molecular diameter, respectively, which are determined to be $\varepsilon_{\mathrm{ww}}=1.0$ and $\sigma_{\mathrm{ww}}=0.8$. Therefore, we would ensure that no penetration of fluid particles through the wall would be occurred.
In order to integrate the equations of motion, we employ the Verlet algorithm ${ }^{16)}$ and the temperature is kept constant using the DPD thermostat ${ }^{17)}$ with friction parameter $\xi=0.5$, Heaviside-type weight functions, and a thermostat cutoff $r_{\mathrm{c}}=2.5 \sigma$. The integration time step $\delta t$ is taken from the following expression:

$$
\delta t=0.01 t_{0}
$$

where $t_{0}$ is our basic time unit and is obtained from the following equation:

$$
t_{0}=\sqrt{\frac{m \sigma^{2}}{48 k_{\mathrm{B}} T}}
$$

where $m$ and $k_{\mathrm{B}} T$ are chosen to be 1 .

Our simulation is restricted to a simple fluid interacting via an LJ potential with $\varepsilon=1.4$ and $\sigma=1.0$. The non-bonded interaction is also given by an LJ potential with $\varepsilon=1.4$ and $\sigma=1.0$. However, the wall-fluid interaction is regarded as a variable parameter, which is given by an LJ potential with strength $\varepsilon_{\mathrm{WL}}$. To reduce the computation time, all interactions are cut off at $r_{\mathrm{c}}=2.5 \sigma$. The total number of fluid particles is 25000, while those forming the tube are 3243 .

To determine the surface tension and viscosity of the LJ fluid, following the Ref. 18) for the LJ fluid (at density $\rho_{1}=0.774$ ), we find that $\eta \approx 6.34 \pm 0.15$. We derive a compatible value for the viscosity by applying an equilibrium MD simulation and then using the correlation function of diagonal pressure tensor components and the standard Kubo relationship. ${ }^{16)}$ From the flat gas-fluid interface observed in the left side of our simulation box (See Fig. 1), it is feasible to estimate the surface tension $\gamma_{\mathrm{lv}}$ from the anisotropy of the pressure tensor components ${ }^{19)}$ as follows:

$$
\gamma_{\mathrm{lv}}=\int\left[p_{z z}(z)-\frac{p_{x x}(z)+p_{y y}(z)}{2}\right] d z
$$

The above equation yields $\gamma_{\mathrm{lv}}=0.735 \pm 0.015$ for the $\mathrm{LJ}$ fluid.

As mentioned previously, the interaction strength between fluid and wall is considered as a variable in our MD simulations. Since it has been proved that this parameter has a crucial influence on the imbibition process at nanoscale. By varying this interaction strength, one can achieve the wetting behavior of the test fluid in a wide range. Finally, we should say that our MD simulations mimic the recent experiments of flow of liquids in nanopores. ${ }^{20)}$

\section{Results and Discussion}

Figure 2 shows the time evolution of the squared height of wetting. It implies that the length of imbibition depends very sensitively on the strength of the wall-fluid interaction. We also summarize the simulation results in Table 1. This Table indicates correlation of the squared length of imbibition as a function of the time for different $\varepsilon_{\mathrm{WL}}$ values in terms of a statistical parameter, namely, the squared correlation coefficient $\left(R^{2}\right)$. The $R^{2}$ statistic is used almost universally in judging regression equations. ${ }^{21)}$ This statistic measures the correlation between the target values and those predicted by a given model. The square of correlation coefficient can take on any value between 0 and 1 , with a value closer to 1 


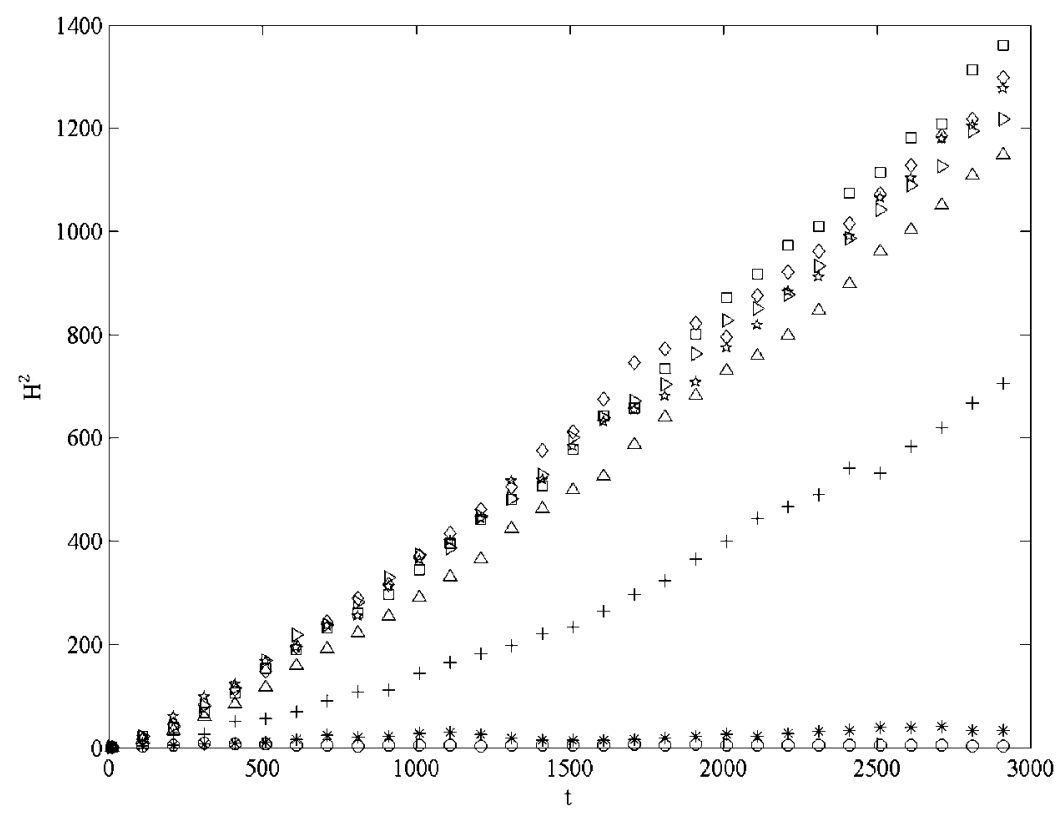

Fig. 2 Plot of the squared height of wetting as a function of the time in the case of imbibition of a designed nanopore by a Lennard-Jones fluid for the wall-fluid interactions $0.6(\bigcirc), 0.8(*), 1.0(+), 1.2(\triangle), 1.4(\square), 1.6(\diamond), 1.8(\lesssim)$ and $2.0(\triangleright)$.

Table 1 Correlation of the squared wetting length as a function of the time in the case of imbibition of a Lennard-Jones fluid into a designed nanopore for various wall-fluid interactions $\left(\varepsilon_{\mathrm{WL}}\right)$ in terms of the squared correlation coefficient $\left(R^{2}\right)$.

\begin{tabular}{ccccccccc}
\hline$\varepsilon_{\mathrm{WL}}$ & 0.6 & 0.8 & 1.0 & 1.2 & 1.4 & 1.6 & 1.8 & 2.0 \\
\hline$R^{2}$ & 0.0090 & 0.6128 & 0.9358 & 0.9758 & 0.9749 & 0.9882 & 0.9813 & 0.9893
\end{tabular}

indicating that the model yields a greater fitness. As can be seen from this Table, for $\varepsilon_{\mathrm{WL}}=0.6$ and 0.8 , a good correlation is not achieved. These cases yield a $R^{2}=$ 0.0090 and 0.6128 , respectively. However, for all other values of $\varepsilon_{\mathrm{WL}}$, a very good relationship between the squared length of imbibition and the time, ranging from $R^{2}=0.9358$ to 0.9893 , is obtained. Incidentally, in the latter $\varepsilon_{\mathrm{WL}}$ values (i.e., $\varepsilon_{\mathrm{WL}}=1.0$ to 2.0 ), the accuracy of the $\mathrm{LW}$ equation is confirmed. Whereas, in the former choices of $\varepsilon_{\mathrm{WL}}$ (i.e., $\varepsilon_{\mathrm{WL}}=0.6$ and 0.8$)$, the simulation results do not obey the simple LW equation and a modification of this equation is needed.

In what follows, we attempt to find a reason for this observed discrepancy between the results of MD simulation and the LW equation. At first, we shall say that the inertia effect in our simulations is small. Since the effect of inertia, which initially proposed by Rideal ${ }^{22}$ and Bosanquet, ${ }^{23)}$ is only significant in the early stages of penetration, or when capillary radius is large. Therefore, we ignore this effect in analysis of the simulation results. In addition, the influence of gravity is also negligible. Since the length of nanotube is sufficiently short to take into consideration this parameter. As stated before, this observed deviation from the LW equation is a result of neglecting the DCA. In order to demonstrate this fact, we shall verify that the MD simulation results are in good agreement with the LW equation considering the DCA (i.e., eq. (3)) where the simple LW equation is not valid (i.e., $\varepsilon_{\mathrm{WL}}=0.6$ and 0.8 ). To this end, we employ the Cox
Table 2 Correlation of the dynamic contact angle as a function of the wetting rate in the case of imbibition of a Lennard-Jones fluid into a designed nanopore for various wall-fluid interactions $\left(\varepsilon_{\mathrm{WL}}\right)$ in terms of the squared correlation coefficient $\left(R^{2}\right)$.

\begin{tabular}{cccc}
\hline$\varepsilon_{\mathrm{WL}}$ & 0.6 & 0.8 & 1.0 \\
\hline$R^{2}$ & 0.9307 & 0.8654 & 0.4574
\end{tabular}

relationship (i.e., eq. (2)) to get the DCA and try to correlate the DCA as a function of the imbibition rate using the MD simulation data. The results of this investigation are summarized in Table 2. As can be seen from this Table, for $\varepsilon_{\mathrm{WL}}=$ 0.6 and 0.8 , one can achieve $R^{2}=0.9307$ and 0.8654 , respectively. This result justifies the use of the modified LW equation along with the Cox equation as a reliable model to describe the kinetics of fluid penetration at nanopores where the simple LW equation tends to break down.

In following section, we would like to show how the precise assessment of our MD simulations leads to a fact regarding the Cox equation. From Fig. 2, it is obvious that for the cases $\varepsilon_{\mathrm{WL}} \geq 1.2$, all simulation data, with a good approximation, fall on the same region. This implies that for those cases, a complete wetting (i.e., $\cos \theta=1$ ) is happened. Since only contact angle varies in the simple LW equation. As a result, one can anticipate a partial wetting of the nanopore for $\varepsilon_{\mathrm{WL}} \leq 1.0$. It was verified that for $\varepsilon_{\mathrm{WL}}=$ 0.6 and 0.8 , the simulation results are in good agreement with the modified LW equation along with the Cox equation. Now, let us suppose that for the case $\varepsilon_{\mathrm{WL}}=1.0$, the contact angle is the dynamic one and obeys the Cox equation. Therefore, we can apply the modified LW equation (i.e. eq. (3)) to this case. The result is given in Table 2. It shows a poor correlation (i.e., $R^{2}=0.4574$ ) indicating the Cox equation is not able to fit the simulation result for this case. To explain why this nonconformity is happened, we shall come back to the theoretical background of this equation. Indeed, this equation 
was developed to deal with only low capillary numbers. Capillary number $C a$ is given by $C a=\eta v / \gamma_{\mathrm{lv}}$, where $v$ is the velocity of imbibition. Hence, this relationship has a drawback to give correct DCA at high capillary numbers. This weakness point of this equation has been also proved experimentally. ${ }^{11)}$ In this case study, it is clear from Fig. 2 that the imbibition rate of nanopore and consequently $\mathrm{Ca}$ has a direct relationship with $\varepsilon_{\mathrm{WL}}$. Moreover, for $\varepsilon_{\mathrm{WL}}=0.6,0.8$ and 1.0, one can calculate $C a=0.0094,0.0223$ and 0.0849, respectively. Now, it is straightforward to understand why the Cox equation is in a very good agreement with the $\varepsilon_{\mathrm{WL}}$ values 0.6 and 0.8 and does not fit the simulation results in the case of $\varepsilon_{\mathrm{WL}}=1.0$. Since in the latter $\varepsilon_{\mathrm{WL}}, C a$ has a greater value compared to the former $\varepsilon_{\mathrm{WL}}$ values.

\section{Conclusions}

MD simulation of imbibition of a simple LJ fluid through a designed nanopore is carried out and the following conclusions are obtained:

(1) Generally, the simple LW equation is a reliable model to explain the kinetics of this phenomenon. However, nonconformity to this equation is observed in some cases. Since the simple LW equation overlooks the significant effect of the DCA on this phenomenon.

(2) A model to derive the DCA (i.e., the Cox equation) is proposed. Among advantages of this equation over other models, one can state that: i) It has the simplest form; ii) It is straightforward to implement; and iii) For the relevant ranges of velocities and contact angles, the Cox correlation agrees with experimental data.

(3) Our MD simulations manifest a flaw of the Cox equation, which is suitable to yield the DCA only at low capillary numbers.

\section{Acknowledgements}

S. Ahadian greatly appreciates Dr. Dimitrov, Professor Milchev, and Professor Binder. In addition, the authors sincerely appreciate the staff of the Center for Computational Materials Science of the Institute for Materials Research (IMR), Tohoku University, for its continuous support of the supercomputing facilities. This work was supported (in part) by Global COE Program "Materials Integration (International Center of Education and Research)", Tohoku University, MEXT, Japan.

\section{REFERENCES}

1) R. Lucas: Kolloid Z. 23 (1918) 15-22.

2) E. W. Washburn: Phys. Rev. 17 (1921) 273-283.

3) S. Supple and N. Quirke: Phys. Rev. Lett. 90 (2003) 214501.

4) S. Supple and N. Quirke: J. Chem. Phys. 122 (2005) 104706.

5) P. G. de Gennes: Rev. Mod. Phys. 57 (1985) 827-863.

6) R. G. Cox: J. Fluid Mech. 168 (1986) 169-194.

7) Y. D. Shikhmurzaev: J. Fluid Mech. 334 (1997) 211-249.

8) T. D. Blake and J. M. Haynes: J. Colloid Interface Sci. 30 (1969) 421-423.

9) G. Martic, T. D. Blake and J. De Coninck: Langmuir 21 (2005) 1120111207.

10) M. Bracke, F. De Voeght and P. Joos: Prog. Colloid Polym. Sci. 79 (1989) 142-149.

11) B. Lavi and A. Marmur: Colloids Surf. A: Physicochem. Eng. Aspects 250 (2004) 409-414.

12) B. Lavi, A. Marmur and J. Bachmann: Langmuir 24 (2008) 1918-1923.

13) P. G. de Gennes, X. Hua and P. Levinson: J. Fluid Mech. 212 (1990) 55-63.

14) A. M. Cazabat: Adv. Colloid Interface Sci. 42 (1992) 65-87.

15) D. I. Dimitrov, A. Milchev and K. Binder: Phys. Rev. Lett. 99 (2007) 054501 .

16) M. P. Allen and D. J. Tildesley: Computer Simulation of Liquids, (Clarendon Press, Oxford, 1987).

17) T. Soddemann, B. Dünweg and K. Kremer: Phys. Rev. E 68 (2003) 046702.

18) J. A. Backer, C. P. Lowe, H. C. J. Hoefsloot and P. D. Iedema: J. Chem. Phys. 122 (2005) 154503.

19) J. S. Rowlinson and B. Widom: Molecular Theory of Capillarity, (Clarendon Press, Oxford, 1982).

20) P. Huber, S. Grüner, C. Schäfer, K. Knorr and A. V. Kityk: Eur. Phys. J. ST 141 (2007) 101-105.

21) N. R. Draper and H. Smith: Applied Regression Analysis, (Wiley, 3rd ed., New York, 1998)

22) E. K. Rideal: Phil. Mag. 44 (1922) 1152-1159.

23) C. H. Bosanquet: Phil. Mag. 45 (1923) 525-531. 